\title{
Role of capsaicin sensitive nerves in epidermal growth factor effects on gastric mucosal injury and blood flow
}

\author{
J Y Kang, C H Teng, F C Chen, A Wee
}

\begin{abstract}
Background-Epidermal growth factor (EGF) and capsaicin protect against experimental gastric mucosal injury. Capsaicin exerts its gastroprotective effect by stimulating afferent neurones leading to release of calcitonin gene related peptide (CGRP) which causes gastric hyperaemia. EGF also causes gastric hyperaemia but whether it acts via capsaicin sensitive neurones is unknown.
\end{abstract}

Aims-To assess the influence of: (1) capsaicin desensitisation on EGF effects on gastric mucosal injury and gastric mucosal blood flow; and (2) close arterial infusion of hCGRP ${ }_{8-37}$, a CGRP antagonist, on EGF effects on gastric mucosal blood flow.

Methods-The absolute ethanol induced gastric mucosal injury model in the rat was used. Gastric mucosal damage was assessed by planimetry and light microscopy. Gastric mucosal blood flow was measured by laser Doppler flowmetry in a gastric chamber preparation.

Results-Capsaicin desensitisation abolished the gastroprotective and gastric hyperaemic effects of EGF. Close arterial infusion of $\mathbf{h C G R P}_{8-37}$ antagonised the hyperaemic effect of both capsaicin and EGF.

Conclusion-Results show that EGF may exert its gastroprotective and gastric hyperaemic effects via capsaicin sensitive afferent neurones.

(Gut 1998;42:344-350)

Division of

Gastroenterology,

Department of

Medicine

J Y Kang

C H Teng

Department of Physiology

F C Chen

Department of Pathology, National University of Singapore

A Wee

Correspondence to: Dr J Y Kang, James Paget Hospital, Lowestoft Road, Gorleston, Great Yarmouth, Norfolk NR31 6LA, UK

Accepted for publication 5 September 1997
Keywords: capsaicin; epidermal growth factor; gastric mucosal injury; gastric mucosal blood flow; calcitonin gene related peptide antagonist; rat

Epidermal growth factor (EGF) is a polypeptide containing 53 amino acids which was first isolated from the mouse submaxillary gland. ${ }^{1}$ It has been shown to protect the gastric mucosa against injury induced by stress or various necrotising agents such as absolute ethanol, hypertonic saline, and aspirin. ${ }^{2}$ EGF also enhances healing of experimental peptic ulcer. ${ }^{3}$ The mechanism of its gastroprotective action is unclear but a trophic effect on gastroduodenal mucosa ${ }^{4}$ enhancement of mucus production, ${ }^{5}$ and increase in gastric mucosal blood flow ${ }^{6}$ are possibilities. The cytoprotective effect of EGF is not mediated by mucosal prostaglandins and is independent of its antisecretory action. ${ }^{7}$
Capsaicin, the pungent ingredient of chilli pepper, is protective against experimental gastric mucosal injury in animals ${ }^{8-10}$ and humans. ${ }^{11}$ It has also been recently shown to enhance healing of experimental gastric ulcer. ${ }^{12}$ It is thought to act via stimulation of afferent neurones causing release of calcitonin gene related peptide (CGRP) which in turn induces gastric hyperaemia with the involvement of nitric oxide. ${ }^{13}{ }^{14}$ Prior capsaicin desensitisation therefore abolishes the gastroprotective effect of capsaicin. The gastric hyperaemic effect of capsaicin is blocked by both capsaicin desensitisation and close arterial infusion of hCGRP $_{8-37}{ }^{13}$ The relation between capsaicin desensitisation and close arterial infusion of hCGRP $_{8-37}$, an antagonist of CGRP, on the one hand, on gastroprotection afforded by EGF and the gastric hyperaemic effect of EGF, on the other hand, has not been well studied.

There is indirect epidemiological evidence to suggest the involvement of both EGF and capsaicin in human peptic ulcer disease. The present study examined interactions between EGF and capsaicin on absolute ethanol induced gastric mucosal injury. In some experimental systems sialoadenectomy increased gastric mucosal injury which is reversed by exogenous EGF. ${ }^{15}{ }^{16}$ We have therefore investigated the effect of EGF in intact, sialoadenectomised, and capsaicin desensitised rats on ethanol induced gastric mucosal injury. We examined the influence of EGF on gastric mucosal blood flow in rats with intact innervation as well as in capsaicin desensitised rats. EGF and capsaicin were also given concomitantly to evaluate their combined effect. hCGRP $_{8-37}$, was administered by close arterial perfusion to determine its effect on EGF induced gastric hyperaemia.

\section{Methods}

ANIMALS AND REAGENTS

Male Sprague-Dawley rats weighing approximately $250 \mathrm{~g}$ were used. They were acclimatised in the animal house for at least a week before sialoadenectomy or sham operation. Capsaicin (Sigma) was dissolved in a solvent consisting of $10 \%$ ethanol, $10 \%$ Tween 80 (Sigma), and $80 \%$ normal saline $(0.15 \mathrm{~N}$ sodium chloride) volume for volume. EGF (Sigma) was dissolved in $0.15 \mathrm{~N}$ sodium chloride. hCGRP ${ }_{8-37}$ (Sigma) was dissolved in $0.1 \%$ bovine serum albumin in saline. The Clinical Research Centre (CRC) cocktail consisted of $1 \mathrm{ml}$ Hypnorm (Janssen, Beerse) containing $0.315 \mathrm{mg}$ fentanyl and $10 \mathrm{mg}$ fluanisone, and 
$1 \mathrm{ml}$ midazolam (Roche, Basle) containing 5 $\mathrm{mg}$ dormicum in $2 \mathrm{ml}$ distilled water.

SIALOADENECTOMY AND DESENSITISATION

After acclimatisation the animals underwent either sialoadenectomy or sham procedure under CRC cocktail anaesthesia $(0.3 \mathrm{ml} / 100 \mathrm{~g})$ and the submandibular glands were removed after ligation of blood vessels and blunt dissection of the closely associated lymph nodes. The animals were allowed to recover from anaesthesia with body temperature kept at $36(0.5)^{\circ} \mathrm{C}$ using a homeothermic control unit. In the sham operation the same procedure was performed except that the glands were inspected but not removed. Ten days after sialoadenectomy or sham operation, the rats received subcutaneous injections of capsaicin or capsaicin solvent. This was performed over two days, with $25 \mathrm{mg} / \mathrm{kg}$ of capsaicin in the morning and $50 \mathrm{mg} / \mathrm{kg}$ in the afternoon on the first day and $50 \mathrm{mg} / \mathrm{kg}$ in the morning on the second day. The capsaicin was dissolved in solvent at a concentration of $10 \mathrm{mg} / \mathrm{ml}$. Control rats received subcutaneous injections of the same volumes of solvent. The effectiveness of capsaicin treatment was checked by instilling a drop of capsaicin solution $(10 \mathrm{mg} / \mathrm{ml}$ capasicin solvent diluted 100 times with saline) into one eye of each rat. Inadequate capsaicin desensitisation would be manifested by instant response of the animal with wiping movements to the eyes. In the present experiment, capsaicin treatment was effective in all the rats treated and no wiping movements were observed.

GASTRIC CHAMBER PREPARATION

A gastric chamber technique, first described by Mersereau and Hinchey, ${ }^{17}$ was used to study gastric mucosal blood flow. Male SpragueDawley rats weighing approximately $200 \mathrm{~g}$ were used. They were acclimatised in the animal house for one week prior to the study and deprived of food for 24 hours prior to the experiment but allowed free access to water. Each animal was anaesthetised with the CRC cocktail. This was given intraperitoneally at a dose of $0.3 \mathrm{ml} / 100 \mathrm{~g}$ which was repeated as necessary. The body temperature was maintained at $36(0.5)^{\circ} \mathrm{C}$ using a homeothermic blanket (Harvard, Oldham, UK) throughout the experiment. Laparotomy was performed through a midline epigastric incision. The stomach was exposed and brought to the abdominal surface by gentle traction. It was then drawn through the centre hole of a plastic platform placed over the animal, opened along the greater curvature, mucosal side uppermost, and the edges pinned out. A plastic rim was then applied and tightened down. An ex vivo gastric chamber was thus made with the major vasculature left intact. The glandular mucosa in the chamber was washed with three changes of normal saline ( $0.15 \mathrm{~N}$ sodium chloride) and bathed with another $1.5 \mathrm{ml}$ of normal saline. Blood pressure was monitored via the carotid artery by a blood pressure transducer (7016 Ugo Basile, Comerio-Varese, Italy) which was calibrated with a mercury manometer and blood pressure transducer calibrator (2900
Ugo Basile, Comerio-Varese, Italy) and recorded with a one channel recorder (7050 Ugo Basile, Comerio-Varese, Italy).

\section{BLOOD FLOW MEASUREMENT}

Gastric mucosal blood flow was measured by a laser Doppler flowmeter (PF2B, Perimed, Sweden) and recorded by the Perisoft program (Perimed, Sweden). The frequency was 12 $\mathrm{KHz}$, the gain one, and the time constant 0.2 seconds. The zero value was defined by placing the laser probe against a white board. The probe was placed just above and perpendicular to the mucosal surface and its position fixed by a metal stand. Measurements were made continuously and at the same site at the anterior wall of the glandular part of the stomach. The readings were transferred on to floppy disks for subsequent analysis.

\section{EXPERIMENTAL PROTOCOL}

Ethanol induced injury

The animals were used for the experiment proper 10 days after subcutaneous treatment with capsaicin or solvent. The 128 rats were already divided into four groups of 32 , which received: sham operation followed by administration of solvent; sham operation followed by administration of capsaicin; sialoadenectomy followed by administration of solvent; or sialoadenectomy followed by subcutaneous injection of capsaicin.

The animals were fasted for 24 hours before the experiment proper but allowed free access to water. They were then given by gavage either $1 \mathrm{ml}$ of solvent, capsaicin $5 \mathrm{mg}$ in $1 \mathrm{ml}$ of solvent, EGF $25 \mu \mathrm{g}$ in $1 \mathrm{ml}$ of saline, or combined capsaicin $5 \mathrm{mg}$ in $0.5 \mathrm{ml}$ of solvent plus EGF $25 \mu \mathrm{g}$ in $0.5 \mathrm{ml}$ saline. Thirty minutes later they were given by gavage $2 \mathrm{ml}$ absolute ethanol. After another hour, the animals were sacrificed.

\section{Gastric mucosal blood flow}

Thirty two capsaicin desensitised animals and 32 control animals were studied in groups of eight. After a stabilising period of 30 minutes, recording of blood flow was begun and continued for another 135 minutes. Basal blood flow was recorded for 45 minutes. For each group one of the following was introduced into the chamber at the end of the basal period: $1.5 \mathrm{ml}$ normal saline; $1.5 \mathrm{ml}$ normal saline plus $5 \mathrm{mg}$ capsaicin in $1 \mathrm{ml}$ solvent given subcutaneously; $25 \mu \mathrm{g}$ EGF in $1.5 \mathrm{ml}$ normal saline; or $25 \mu \mathrm{g}$ EGF in $1.5 \mathrm{ml}$ normal saline plus $5 \mathrm{mg}$ capsaicin in $1 \mathrm{ml}$ solvent given subcutaneously. Each solution was removed at the end of 15 minutes and replaced with normal saline.

In most studies on capsaicin gastroprotection capsaicin was given by gavage. We have, however, previously shown that subcutaneous administration has the same gastroprotective effect as intragastric administration on absolute ethanol induced gastric mucosal injury. ${ }^{10}$ In the present studies on gastric mucosal blood flow, therefore, we administered capsaicin subcutaneously rather than intragastrically to avoid any possible interaction with EGF. 
Effect of concomitant hCGRP $P_{8-37}$ administration Animals with intact innervation were used. For each rat a gastric chamber was prepared as described earlier. The abdominal aorta was exposed and a $24 \mathrm{G} \times 3 / 4$ inch over the needle catheter (Terumo, Tokyo, Japan) was introduced into the aorta in a retrograde fashion until the tip of the cannula lay just above the origin of the coeliac artery. Close arterial infusion of hCGRP ${ }_{8-37}(500 \mathrm{pmol} / \mathrm{kg} / \mathrm{min})$ or vehicle (bovine serum albumin $0.1 \%$ in saline) was started at $10 \mu \mathrm{l} /$ minute. One group of animals received subcutaneous injections of capsaicin 5 $\mathrm{mg}$. Another group received $25 \mu \mathrm{g}$ EGF in 1.5 $\mathrm{ml}$ normal saline applied directly into the gastric chamber. The control and capsaicin groups received $1.5 \mathrm{ml}$ normal saline into the gastric chamber.

\section{ASSESSMENT OF MUCOSAL DAMAGE}

Macroscopic

After each animal was killed by cervical dislocation the abdomen was quickly opened and the stomach exposed after ligating the oesophagus. Gastric juice was collected via an incision in the duodenum and $5 \mathrm{ml} \mathrm{10 \%} \mathrm{neu-}$ tral buffered formalin was instilled into the stomach via the incision. The stomach was then opened along the greater curvature, fixed in formalin overnight, and photographed.

The amount of macroscopic damage was measured by a digital planimeter (Planix 7, Tamaya Technic Incorporated, Japan) and expressed as a percentage of whole total glandular area. The person assessing the damage was unaware of the group to which each animal belonged.

Table 1 Ethanol induced gastric mucosal damage: the effect of sensory ablation and sialoadenectomy. Macroscopic assessment (mean (SEM) \% damage)

\begin{tabular}{|c|c|c|}
\hline & Intact innervation & Sensory ablation \\
\hline \multicolumn{3}{|l|}{ Sham operation } \\
\hline Solvent & $26.7(5.6)$ & $22.2(3.2)$ \\
\hline EGF & $8.4(1.6)^{\star}$ & $33.6(8.7) \dagger$ \\
\hline Capsaicin & $0.2(0.2)^{\star}$ & $19.6(3.2) \dagger$ \\
\hline Capsaicin + EGF & $0.5(0.2)^{\star}$ & $24.0(8.9) \dagger$ \\
\hline \multicolumn{3}{|l|}{ Sialoadenectomy } \\
\hline Solvent & $25.3(6.9)$ & $26.6(2.8)$ \\
\hline EGF & $16.1(3.2)$ & $31.4(5.8)$ \\
\hline Capsaicin & $0.8(0.6)^{\star}$ & $26.0(9.1) \dagger$ \\
\hline Capsaicin + EGF & $2.2(1.6)^{\star}$ & $27.0(6.7) \dagger$ \\
\hline \multicolumn{3}{|c|}{$\begin{array}{l}{ }^{\star} \mathrm{p}<0.05 \text { versus respective solvent groups; } t \mathrm{p}<0.05 \text { versus } \\
\text { respective intact innervation groups. }\end{array}$} \\
\hline \multicolumn{3}{|c|}{$\begin{array}{l}\text { Table } 2 \text { Ethanol induced gastric mucosal damage: the } \\
\text { effect of sensory ablation and sialoadenectomy. Microscopic } \\
\text { assessment (grade } 3 \text { damage; mean (SEM) \%) }\end{array}$} \\
\hline & Intact innervation & Sensory ablation \\
\hline \multicolumn{3}{|l|}{ Sham operation } \\
\hline Solvent & $27.5(3.4)$ & $21.3(4.2)$ \\
\hline EGF & $10.7(3.6)^{\star}$ & $27.5(7.2) \dagger$ \\
\hline Capsaicin & $4.3(2.1)^{\star}$ & $32.5(3.7) \dagger$ \\
\hline Capsaicin + EGF & $7.5(3.1)^{\star}$ & $28.2(9.0) \dagger$ \\
\hline \multicolumn{3}{|l|}{ Sialoadenectomy } \\
\hline Solvent & $25.1(6.8)$ & $33.8(3.5)$ \\
\hline EGF & $16.0(4.1)$ & $36.5(5.4) \dagger$ \\
\hline Capsaicin & $2.5(2.5)^{\star}$ & $31.7(5.7) \dagger$ \\
\hline Capsaicin + EGF & $7.8(3.1)^{\star}$ & $25.5(4.5) \dagger$ \\
\hline
\end{tabular}

$\star_{\mathrm{p}}<0.05$ versus respective solvent groups; $\uparrow \mathrm{p}<0.05$ versus respective intact innervation groups.
Light microscopy

After fixation, each stomach was divided into two and each half subdivided into four tissue strips by cutting along the whole width of the half stomach. The blocks were than embedded in paraffin wax. Histological sections $5 \mu \mathrm{m}$ thick were cut in a standard fashion and stained with haematoxylin and eosin. Tissue damage was evaluated using a modification of Lacy and Ito's classification ${ }^{1018}$ as follows: grade 0 - normal surface epithelial cells, gastric pits, and glands; grade 1-damage to surface epithelial cells without damage to the cells lining the gastric pits; grade 2 -in addition to extensive luminal surface cell damage the cells lining the gastric pits were also disrupted and exfoliated, but the gastric gland cells were not damaged; grade 3-cellular damage was evident in the gastric glands. The span of each degree of injury was measured microscopically with an ocular micrometer. The person evaluating the injury was unaware of the group to which each rat belonged.

In microscopic assessment of gastric mucosal damage, the most significant effects with both capsaicin and EGF were observed for reduction of deep mucosal damage (grade 3 ). In this report, therefore, only data for grade 3 damage will be presented.

\section{STATISTICAL CONSIDERATIONS}

All results are given as mean (SEM). For the blood flow study, the first reading of gastric blood flow was taken as the basal blood flow. Subsequent readings of blood flow taken at 15 minute intervals were expressed as percentages of the basal blood flow. The areas under the curve were also calculated for the 90 minute period after exposure to the study substance. Statistical analysis was performed using the Statistical Package for Social Sciences for Windows (SPSSWIN). One way analysis of variance was performed to detect statistical differences between the groups. For the blood flow studies the same procedure was repeated for the different groups at each time point. For percentage values, angular or arcsin transformation of the data was carried out. ${ }^{192}$ This transformation was developed for the analysis of binomial data expressed as percentages and is recommended when the percentages cover a wide range of values. Where treatment of data requiring non-parametric techniques was needed, the variables were ranked and analyses performed on the ranked data. This was to avoid the use of the non-parametric KruskalWallis test which yields a $\chi^{2}$ statistic with one degree of freedom. Analysis of variance on ranked data gives a more rigorous test as it uses the $\mathrm{F}$ test. ${ }^{21}$ Probability values less than 0.05 were considered significant.

\section{Results}

ETHANOL INDUCED DAMAGE

Tables 1 and 2 present ethanol induced damage in the different groups. 

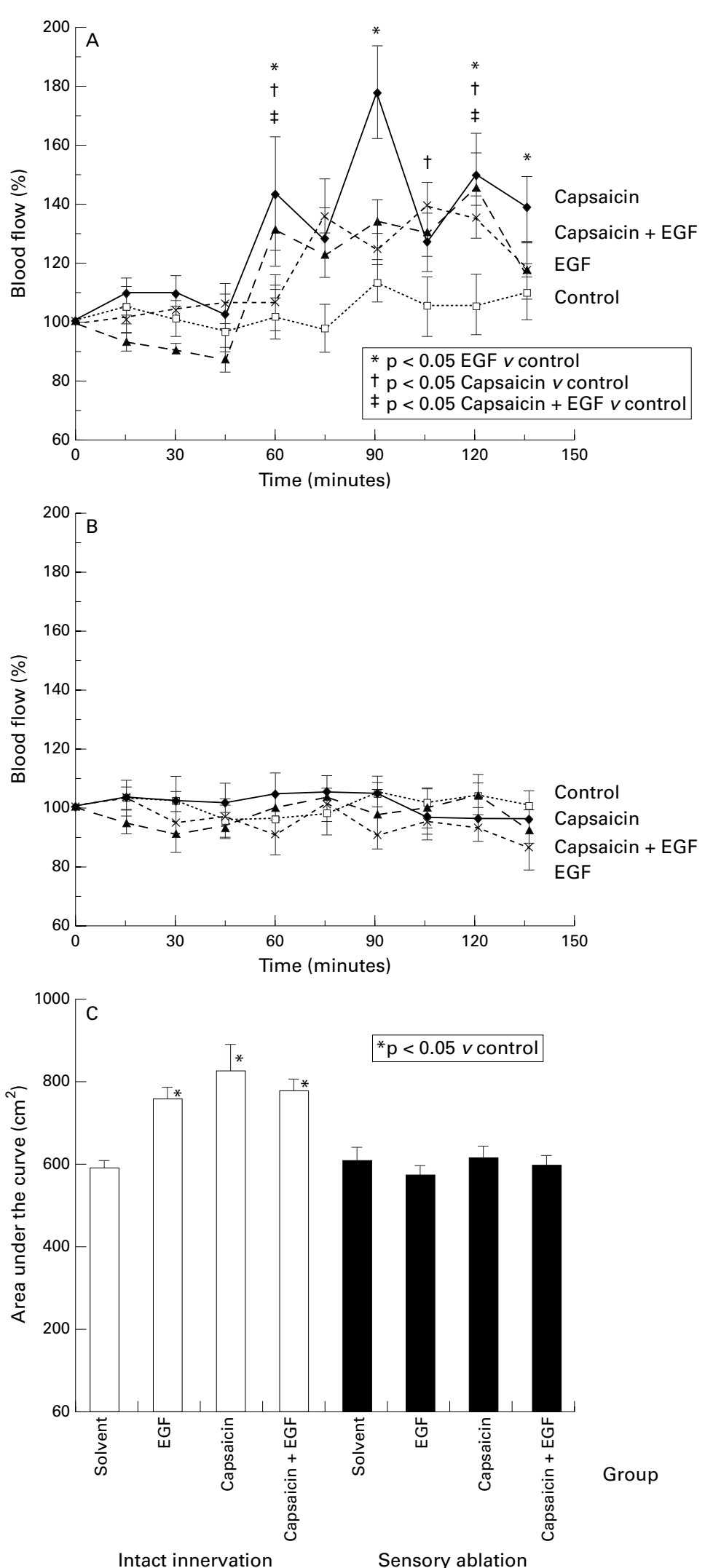

Figure 1 Effect of EGF, capsaicin, and their combination on gastric mucosal blood flow. (A) Animals with intact innervation; (B) capsaicin desensitised animals; (C) data shown as areas under the curve. Results are expressed as mean (SEM), $n=8$ per group.
Effect of capsaicin and EGF on animals with intact innervation and salivary glands

Preadministration of both EGF and capsaicin reduced subsequent ethanol induced gastric mucosal injury in terms of macroscopic and grade 3 microscopic damage. Combined use of both EGF and capsaicin did not yield any further advantage compared with the use of either agent alone.

\section{Effect of capsaicin desensitisation}

Capsaicin desensitisation totally abolished the protective effect of pretreatment with EGF, capsaicin, or capsaicin combined with EGF. However, capsaicin desensitisation did not increase absolute ethanol induced gastric mucosal injury in the solvent group whether sialoadenectomy or sham operation had taken place.

\section{Effect of sialoadenectomy}

Compared with sham operation, sialoadenectomy had no effect on the amount of ethanol induced injury whether pretreatment was with solvent, EGF, capsaicin, or capsaicin and EGF combined. This was the case whether or not prior capsaicin desensitisation had taken place. In this experimental model, therefore, sialoadenectomy did not influence the development of gastric mucosal injury, nor of gastroprotection afforded by EGF or capsaicin.

\section{Effect of both sialoadenectomy and capsaicin desensitisation}

In animals which had undergone both sialoadenectomy and capsaicin desensitisation there was no increase in ethanol induced damage after pretreatment with solvent when compared with animals with intact innervation which had undergone sialoadenectomy, those which had undergone capsaicin desensitisation and sham operation, or those with intact innervation who underwent sham operation. No protection was seen with pretreatment with capsaicin, EGF, or a combination of the two agents.

GASTRIC MUCOSAL BLOOD FLOW

Figure 1 presents the effects of the different treatments on gastric mucosal blood flow.

Capsaicin desensitisation abolished the hyperaemic effect of both capsaicin and EGF. In animals with intact innervation, topical application of capsaicin increased gastric mucosal blood flow. EGF led to a similar hyperaemic response but the combination of EGF and capsaicin in the doses used did not increase the blood flow beyond that caused by either drug alone. In contrast, rats in whom capsaicin sensitive afferent neurones had been ablated did not have a gastric hyperaemic response to capsaicin, EGF, or their combination. The areas under the curve over the 90 minute period were 594.8 (20.9) for the solvent group, 760.2 (33.6) for the EGF group ( $p<0.05$ with respect to solvent), 829.8 (64.5) for the capsaicin group ( $p<0.05$ with respect to solvent), and 775.9 (31.5) $\mathrm{cm}^{2}$ for the combined capsaicin and EGF group $(p<0.05$ with respect to solvent) in animals with intact innervation. For 

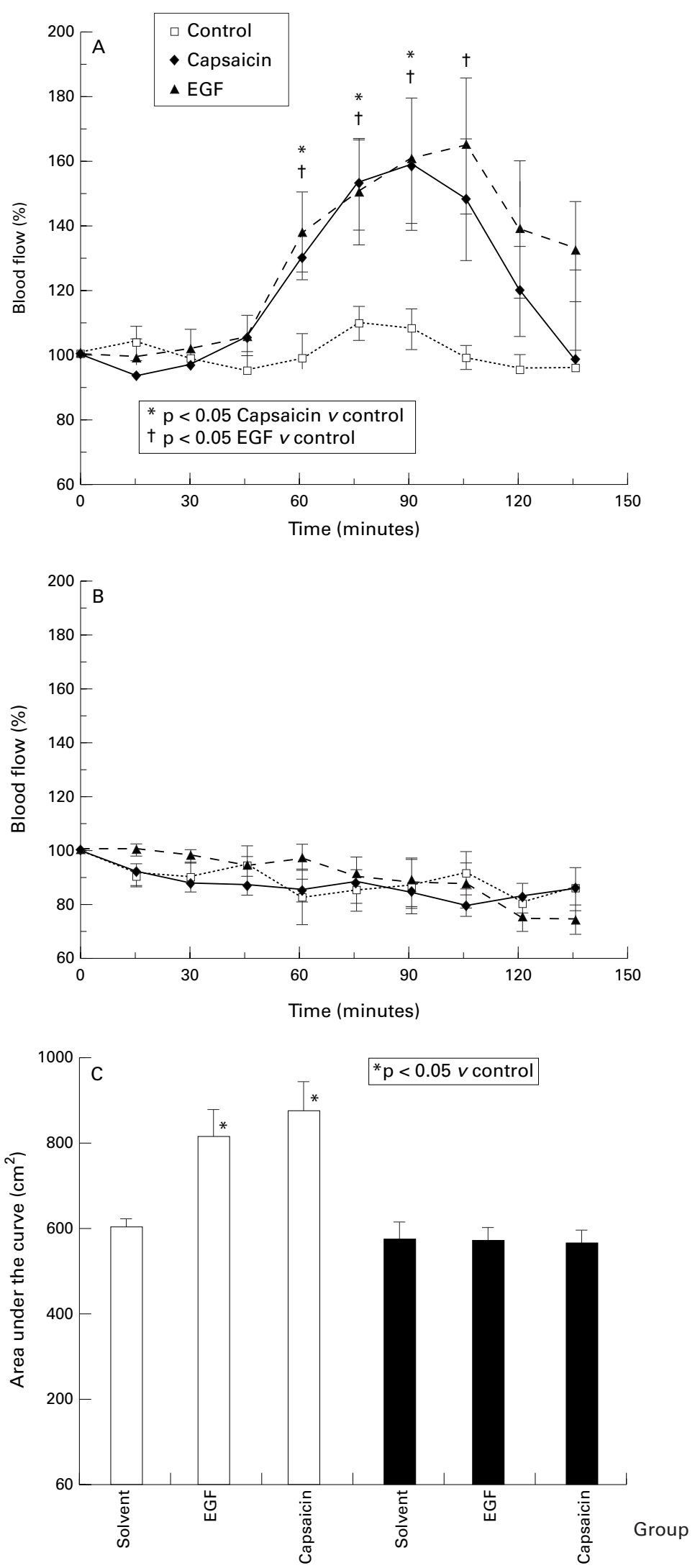

Vehicle

hCGRP 8-37

Figure 2 Effect of close arterial infusion of $h C G R P_{8-37}$ on the gastric hyperaemic response to EGF and capsaicin. (A) Vehicle; (B) concomitant infusion of hCGRP ${ }_{8-37}$ (500 pmol/kg/min); (C) data shown as areas under the curve. capsaicin desensitised rats the areas under the curve for the corresponding groups were: 608.1 (32.5), 571.4 (22.7), 610.0 (29.8), and 588.5 (24.4) $\mathrm{cm}^{2}$ (NS). Arterial blood pressure was maintained throughout all experiments and there were no significant differences in blood pressure between the groups.

EFFECT OF CONCOMITANT ADMINISTRATION OF hCGRP $_{8-37}$

Figure 2 presents the effects of $\mathrm{hCGRP}_{8-37}$ on blood flow.

Close arterial infusion of $\mathrm{hCGRP}_{8-37}$ concomitantly with administration of EGF or capsaicin completely abolished the hyperaemic effect of each substance. In contrast, close arterial infusion of $\mathrm{hCGRP}_{8-37}$ by itself had no significant effect on gastric mucosal blood flow. The areas under the curve for the 90 minute study period were 605.4 (18.9), 812.5 (61.3) $(\mathrm{p}<0.05)$, and $863.2(67.3) \mathrm{cm}^{2}(\mathrm{p}<0.05)$ in the solvent, EGF, and capsaicin groups, respectively, in the control experiment and 565.3 (45.1), 556.7 (33.3), and 549.2 (27.7) $\mathrm{cm}^{2}$ (NS) for the same groups when hCGRP ${ }_{8-37}$ was administered concomitantly. Arterial blood pressure recordings were performed throughout the studies and no significant differences were seen between the groups.

\section{Discussion}

Various clinical and epidemiological observations suggest the involvement of EGF and capsaicin in human peptic ulcer disease. In humans salivary secretion of EGF is decreased in rheumatoid disease. ${ }^{22}$ This has been suggested as one possible explanation for the purported susceptibility of these patients to gastric ulceration. ${ }^{22}$ A lower frequency of peptic ulceration in northern Indians compared with southern Indians has been linked to a high fibre diet which requires more mastication and saliva production. ${ }^{2324} \mathrm{~A}$ high fibre diet has also been shown to reduce relapse rates in duodenal ulcer $^{25}{ }^{26}$ but pectin, a fibre which does not require mastication, had no effect. ${ }^{27}$ In Singapore, Malays and Indians, who consume more chilli than the Chinese, are less susceptible to peptic ulcer disease. ${ }^{28}$ Racial differences in the incidence of perforated peptic ulcer decreased over three decades confirming the involvement of environmental factors such as diet. ${ }^{29}$ Among Chinese subjects peptic ulcer patients had consumed less chilli than controls, even after allowance was made for avoidance of chilli due to dyspepsia. ${ }^{30}$

In our hands sialoadenectomy did not increase subsequent ethanol induced gastric mucosal damage. Konturek et al had shown earlier that sialoadenectomy increased subsequent stress induced gastric mucosal damage. ${ }^{15}$ Removal of submandibular and sublingual glands together with ligation of the parotid gland ducts also enhanced bile salt induced gastric mucosal damage. ${ }^{16}$ Konturek et al, however, also found that sialoadenectomy had no effect on gastric mucosal damage produced by absolute ethanol, ${ }^{31}$ consistent with the present results. It could be that gastric mucosal injury induced by absolute ethanol is too severe for a 
weak gastroprotective effect of endogenous EGF to be detected.

Holzer et $a l^{2}$ reported an increase in gastric mucosal damage produced by $15 \%$ and $25 \%$ ethanol after afferent nerve desensitisation by capsaicin. In experiments by Uchida et al however, capsaicin desensitisation did not enhance absolute ethanol induced gastric mucosal damage. ${ }^{9}$ Our results are similar to those of Uchida et al. The discrepancy may again relate to differences in the concentration of ethanol used.

Capsaicin is thought to exert its gastroprotective effects by stimulating sensory neurones in the stomach which in turn leads to gastric hyperaemia. This hyperaemic effect is mediated by CGRP which is released by capsaicin from afferent nerve endings. ${ }^{33}$ Thus close arterial infusion of CGRP at the same dose which stimulates gastric mucosal blood flow prevented $25 \%$ ethanol and aspirin induced gastric mucosal injury. ${ }^{34} \mathrm{hCGRP}_{8-37}$, an antagonist of CGRP, abolished the gastroprotective effect of capsaicin on $50 \%$ ethanol and indomethacin induced gastric mucosal injury. ${ }^{1435}$

The underlying pathophysiological mechanism for gastroprotection by EGF has not been elucidated but an increase in gastric mucosal blood flow has been suggested as one possibility. ${ }^{6}$ Two recent studies, so far only reported in abstract form, examined the relation between capsaicin desensitisation and the gastroprotective and gastric hyperaemic effects of EGF. Stroff and Peskar ${ }^{36}$ found that both capsaicin desensitisation and hCGRP ${ }_{8-37}$ infusion suppress the protective effect of intravenous urogastrone, a source of EGF, on absolute ethanol induced gastric mucosal injury. Pretreatment with a nitric oxide synthase blocker to prevent gastric hyperaemia also counteracted the protection by urogastrone. Brzozowski et $a b^{37}$ reported that the rise in EGF content of gastric mucosa after stress was reduced by capsaicin desensitisation. The present study shows that both the gastroprotective and gastric hyperaemic effects of EGF are lost following ablation of capsaicin sensitive afferent neurones. It may therefore be that EGF protects the gastric mucosa in the same way as capsaicin, via stimulation of capsaicin sensitive afferent fibres, which in turns leads to release of CGRP with resultant gastric hyperaemia. This hypothesis is supported by our findings on gastric mucosal blood flow and by our demonstration that close arterial infusion of $\mathrm{hCGRP}_{8-37}$ abolished the hyperaemic response to EGF.

The interaction between EGF and capsaicin is of interest for three reasons. Firstly, it may help in the understanding of underlying pathophysiological mechanisms. Secondly, it may explain epidemiological observations regarding peptic ulcer frequency in countries where chilli is widely used. Thirdly, as both EGF and capsaicin have the potential to be used as therapeutic agents in peptic ulcer disease any synergistic effect may be important. Our results show no interaction between sialoadenectomy and capsaicin desensitisation in the develop- ment of absolute ethanol induced gastric mucosal injury, nor in the gastroprotective effect of EGF and capsaicin. A different experimental model, or dose response studies may have to be used to confirm or exclude such an effect. The study on gastric mucosal blood flow also showed no additive effect with concurrent use of EGF and capsaicin.

One drawback of the present study is the use of absolute ethanol, which causes a particularly severe form of gastric mucosal injury. It is possible that we are looking here at pharmacological rather than physiological actions of EGF. Lower concentrations of ethanol may have allowed interaction between EGF and capsaicin as well as the effects of sialoadenectomy and capsaicin desensitisation to be shown better. Future studies should also include the use of different doses of EGF and capsaicin.

Playford et $a l^{8}$ have recently shown that the use of hypnorm anaesthesia affected the degree of gastric mucosal injury due to non-steroidal anti-inflammatory drugs. Absolute ethanol induced injury as used in the present study is, however, acid independent. Also, any effect of the anaesthetic on gastric mucosal damage would have been seen in both the control and experimental groups.

In conclusion, our results suggest, firstly, that the gastroprotective effect of EGF may be secondary to its effect on gastric mucosal blood flow and, secondly, that EGF may exert these effects via stimulation of capsaicin sensitive afferent neurones with release of CGRP. No interaction could be shown between sialoadenectomy and sensory ablation in gastroprotection by either capsaicin or EGF against absolute ethanol induced injury. We were also unable to find any synergistic effect between capsaicin and EGF on either gastroprotection or gastric mucosal blood flow in this model.

This work was supported by Research Grant RP900309, National University of Singapore, as well as GR $06085 \mathrm{H}$ generously donated by the Shaw Foundation. We would like to thank Associate Professor K C Lun for statistical advice and Associate Professor K O Lee for his helpful comments. This study was presented at the Autumn 1995 meeting of the British Society of Gastroenterology.

1 Savage CR Jr, Inagami T, Cohen S. The primary structure of epidermal growth factor. $\mathcal{F}$ Biol Chem 1972;247:7612-21

2 Konturek SJ. Role of epidermal growth factor in gastro protection and ulcer healing. Scand F Gastroenterol 1988;23: 129-33.

3 Olsen PS, Poulsen SS, Therkelsen K, Nexo E. Oral administration of synthetic human urogastrone promotes healing of chronic duodenal ulcer in rats. Gastroenterology 1986;90: 911-7.

4 Dembinski A, Gregory H, Konturek SJ, Polanski M. Trophic action of epidermal growth factor on the pancreas
and gastroduodenal mucosa in rats. F Physiol 1982;325:3542 .

5 Kelly SM, Hunter JO. Epidermal growth factor stimulates synthesis and secretion of mucus glycoproteins in human gastric mucosa. Clin Sci 1990;79:425-7.

6 Hui WM, Chen BW, Kung AWC, Cho CH, Luk CT, Lam SK. Effect of epidermal growth factor on gastric blood flow in rats: possible role in mucosal protection. Gastroenterology 1993;104:1605-10.

7 Konturek SJ, Radecki T, Brzozowski T, et al. Gastric cytoprotection by epidermal growth factor. Role of endogenous prostaglandin and DNA synthesis. Gastroenterology $1981 ; 81: 438-43$.

8 Holzer P, Lippe ITh. Stimulation of afferent nerve endings by intragastric capsaicin protects against ethanol-induced damage of gastric mucosa. Neuroscience 1988;27:981-7.

9 Uchida $M$, Yano S, Watanabe K. The role of capsaicinsensitive afferent nerves in protective effect of capsaicin against absolute ethanol-induced gastric lesions in rats. $f p n$ fPharmacol 1991;55:279-82. 
10 Kang JY, Teng CH, Wee A, Chen FC. The effect of capsaicin and chilli on ethanol-induced gastric mucosal injury in cin and chilli on ethanol-ind

11 Yeoh KG, Kang JY, Yap I, et al. Chilli protects against aspirin-induced gastroduodenal mucosal injury in humans. Dig Dis Sci 1995;40:580-3.

12 Kang JY, Teng CH, Chen FC. Effect of capsaicin and cimetidine on the healing of acetic acid induced gastric ulcer in the rat. Gut 1996;38:832-6.

13 Li DS, Raybould HE, Quintero E, Guth PH. Role of calcitonin gene-related peptide in gastric hyperaemic response to intragastric capsaicin. Am f Physiol 1991;261:G657-61.

14 Lamberecht N, Burchert M, Respondek M, Muller KM Peskar BM. Role of calcitonin gene-related peptide and nitric oxide in the gastroprotective effect of capsaicin in the rat. Gastroenterology 1993;104:1271-80.

15 Konturek SJ, Brzozowski T, Konturek PK, Majka J, Dembinski A. Role of salivary glands and epidermal growth factor (EGF) in gastric secretion and mucosal integrity in factor (EGF) in gastric secretion and mucosal int

16 Skinner KA. Teppeman BL. Influence of desalivation on acid secretory output and gastric mucosal integrity in the $181 \cdot 335-9$.

17 Mersereau WA, Hinchey EJ. Effect of gastric acidity on gastric ulceration induced by haemorrhage in the rat, utilising a gastric chamber technique. Gastroenterology 1973;64 1130-5.

18 Lacy ER, Ito S. Microscopic analysis of ethanol damage to rat gastric mucosa after treatment with a prostaglandin. Gastroenterology 1982;83:619-25.

19 Steel RGD, Torrie JH. Principles and procedures of statistics - a biometrical approach. 2nd edn. New York: McGraw-Hill, 1980.

20 Fisher RA, Yates F. Statistical tables for biological, agricultural and medical research. 6th end. Edinburgh: Oliver and Boyd, 1963.

21 SAS Institute Inc. SAS user's guide: statistics. Version 5 edition. North Carolina: SAS Institute Inc., 1985:651.

22 Kelly SM, Crampton J, Hunter JO. Decreased salivary epidermal growth factor in rheumatoid disease: a possible mechanism for increased susceptibility to gastric ulceramechanism for increased susc
tion. BMF 1990;301:422-3.

23 Malhotra SL. Peptic ulcer in India and its aetiology. Gut 1964;5:412-6.

24 Tovey FI. Peptic ulcer in India and Bangladesh. Gut 1979:20:329-47.

25 Malhotra SL. A comparison of unrefined wheat and rice diets in the management of duodenal ulcer. Postgrad Med $f$ 1978;54:6-9.
26 Rydning A, Berstad A, Aadland ER. Prophylactic effect of dietary fibre in duodenal ulcer disease. Lancet 1982;ii: 736-9.

27 Kang JY, Tay HH, Guan R, Math MV, Yap I, LaBrooy SJ. Dietary supplementation with pectin in the maintenance treatment of duodenal ulcer-a controlled study. Scand 7 Gastroenterol 1988;23:95-9.

28 Kang JY, SJ LaBrooy, Yap I, et al. Racial differences in peptic ulcer frequency in Singapore. 7 Gastroenterol Hepatol 1987;2:239-44.

29 Kang JY. Peptic ulcer surgery in Singapore 1951-80 with particular reference to racial differences in incidence. Aust NZ F Med 1985;15:604-8.

30 Kang JY, Yeoh KG, Chia HP, et al. Chilli-protective factor against peptic ulcer? Dig Dis Sci 1995;40:576-9.

31 Konturek SJ, Dembinski A, Warzecha Z, Bielanski W, Brzozowski T, Drozdowicz D. Epidermal growth factor (EGF) in the gastroprotective and ulcer healing actions of colloidal bismuth subcitrate (De-Nol) in rats. Gut 1988;29:894-902.

32 Holzer P, Pabst MA, Lippe Ith, et al. Afferent nervemediated protection against deep mucosal damage in the rat stomach. Gastroenterology 1990;90:838-48.

33 Holzer P, Peskar BM, Peskar BA, Amann R. Release of calcitonin gene-related peptide induced by capsaicin in the vascularly perfused stomach. Neurosci Lett 1990;108:195200.

34 Lippe IT, Lorbach M, Holzer P. Close arterial perfusion of calcitonin gene-related peptide into the rat stomach inhibits aspirin- and ethanol-induced haemorrhagic damage. Regul Pept 1989;26:35-46.

35 Kinoshita Y, Inui T, Chiba T. Calcitonin gene-related peptide: a neuro-transmitter involved in capsaicin-sensitive afferent nerve-mediated gastric mucosal protection. $\mathcal{F}$ Clin Gastroenterol 1993;17(suppl 1):S27-32.

36 Stroff T, Peskar BM. Capsaicin-sensitive afferent nerves, calcitonin gene-related peptide and nitric oxide participate in the gastroprotective effect of epidermal growth factor (urogastrone) in the rat [abstract]. Gastroenterology 1995; 108:A227.

37 Brzozowski T, Konturek SJ, Pytko-Polonczyk J. Capsaicinsensitive neurons interact with epidermal growth factor in healing of acute gastric lesions in rats [abstract]. Gastroenterology 1996;110:A71.

38 Playford RJ, Vesey DA, Haldane S, Alison MR, Calam J. Dose-dependent effects of fentanyl on indomethacininduced gastric damage. Digestion 1991;49:198-203. 